\title{
Control electrónico on/off para el protocolo de temperatura usado en la criopreservación de embriones de ovino
}

\section{Controle eletrônico on/off para protocolo de temperatura utilizado na criopreservação de embriões de ovinos}

DOI: $10.46932 / \mathrm{sfjdv} 2 \mathrm{n} 3-082$

Received in: May 1st, 2021

Accepted in: Jun 30th, 2021

\author{
Héctor Ladislao Huisacaina Soto \\ Ing. Electrónico, Universidad Nacional Agraria La Molina \\ Av. La Molina s/n, Lima, Perú \\ E-mail: hhuisac@lamolina.edu.pe \\ Juan Manuel Pesantes Rojas \\ Lic. Físico, Universidad Nacional Agraria La Molina \\ Av. La Molina s/n, Lima, Perú \\ E-mail: jmpesantes@lamolina.edu.pe \\ Manuel Herrera Ortiz \\ Ing. Zootecnista, Universidad Nacional Agraria La Molina \\ Av. La Molina s/n, Lima, Perú \\ E-mail: m_herrera_o@yahoo.com
}

\section{RESUMEN}

Se describe un sistema de control electrónico diseñado para obtener una temperatura variable en función del tiempo, especificado en los protocolos para la preservación criogénica de embriones. Se usó el vapor del nitrógeno líquido para lograr un enfriamiento gradual y controlado, desde la temperatura del ambiente hasta $-35{ }^{\circ} \mathrm{C}$; el enfriamiento se hizo mediante un circuito electrónico de control ON/OFF, basado en un procesador digital de señales (DSP). Se hicieron pruebas del sistema para obtener la curva temperatura vs. tiempo recomendado por el protocolo de criopreservación de embriones de ovinos; los resultados obtenidos permitieron concluir que el diseño presentado permite controlar adecuadamente la temperatura al interior de la cámara dentro del rango establecido con errores menores a $1^{\circ} \mathrm{C}$.

Palabras clave: Control ON/OFF, criopreservación, Procesador digital de señales.

\begin{abstract}
An electronic control system designed to obtain a time-varying temperature specified in the protocols for cryogenic embryo preservation is described. Liquid nitrogen vapour was used to achieve a gradual and controlled cooling from room temperature to $-35{ }^{\circ} \mathrm{C}$; the cooling was done by means of an electronic ON/OFF control circuit based on a digital signal processor (DSP). The system was tested to obtain the temperature vs. time curve recommended by the protocol for the cryopreservation of ovine embryos; the results obtained allowed the conclusion that the design presented allows the temperature inside the chamber to be adequately controlled within the established range with errors of less than $1^{\circ} \mathrm{C}$.
\end{abstract}

Keywords: ON/OFF control, cryopreservation, digital signal processor. 


\section{INTRODUCCIÓN}

El uso de nitrógeno en fase líquida o gas, da resultados de temperaturas muy bajas, entre $-135^{\circ} \mathrm{C}$ y $-196^{\circ} \mathrm{C}$, que permite la preservación y almacenamiento a corto o largo plazo de materiales biológicos (Lopes et al., 1989). Así por ejemplo, en laboratorios de reproducción animal, se realiza el congelamiento de semen y embriones de bovinos, ovinos, camélidos y otros animales para su posterior uso en reproducción (Staples \& Page, 1987). Existen diferentes protocolos para el congelamiento dependiendo del material biológico que se va a congelar. Los protocolos establecen entre como debe ser la variación de temperatura del material biológico durante su congelamiento, para obtener un óptimo resultado con la supervivencia de la mayor cantidad de células, ya sea de espermatozoides o de embriones. Fonseca et. al (2018), usaron un equipo de congelamiento Freeze Control CL 5500® (Cryologic, Victoria, Australia) en el cual programaron el protocolo de criopreservación para embriones de cabra (Fig. 1), este equipo, por su costo no es de fácil adquisición. Rubio (2001) describe el protocolo para embriones de equino, cuyo protocolo es similar al de la Fig. 1, pero con diferentes intervalos de tiempo. En el presente trabajo se seguirá el protocolo para embriones de ovino descrito por Staples \& Page (1987).

El control electrónico puede ser de diferentes tipos, Salazar Rivera et al. (2021) describen un control termoeléctrico PWM (modulación por ancho de pulso) usado para un sistema de climatización. Moreano et al. (2019) hicieron pruebas de funcionamiento de controladores de temperatura, concluyendo que el controlador ON/OFF entrega buenas respuestas en términos de operación presentando un menor tiempo de estabilización y una mejor estabilidad en estados estables, por esta razón se harán las pruebas con el control de ON/OFF, llamado también todo/nada. De estos dos métodos mencionados arriba, el último es uno de los más sencillo de programar.

Figura 1. Protocolo para criopreservación de embriones de caprinos (Fuente Fonseca et.al).

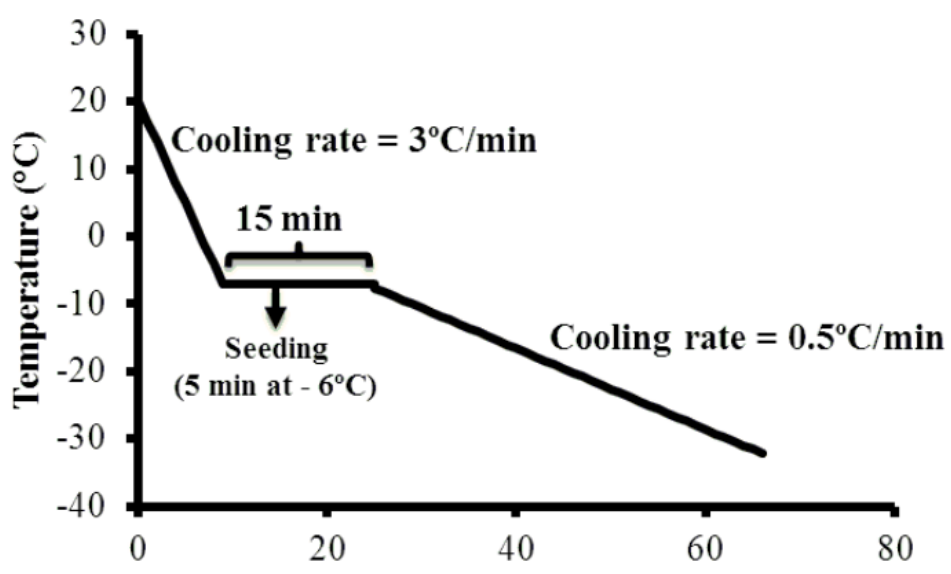

Time (min)

\section{MATERIALES Y MÉTODOS}




\subsection{CÁMARA DE CONGELAMIENTO}

En la figura 2, se muestra el esquema de la cámara de congelamiento, el cual consiste en una caja de un material de baja conductividad térmica que minimice la interacción con la temperatura ambiental, en este caso se ha acondicionado una caja de poliestireno expandido conocido como teknoport, donde se deposita el nitrógeno líquido, se usó aproximadamente 2 litros para una prueba de 2 horas; el nitrógeno líquido hierve aproximadamente a $-196^{\circ} \mathrm{C}$, por lo que a temperatura ambiental se encuentra en ebullición y se producen vapores de nitrógeno a temperaturas de $-196^{\circ} \mathrm{C}$ en puntos cercanos a la superficie líquida del nitrógeno.

En la figura 3 se muestra una vista superior de la cámara de enfriamiento, se puede ver el soporte de rectangular de aluminio y el calefactor 2 en el fondo de la caja. Este calefactor es el que permite incrementar o disminuir los vapores de nitrógeno que rodean al soporte con las muestras biológicas.

Dentro de la cámara se acondicionarán las muestras biológicas que deberán estar contenidas en pajillas (pequeños tubos de $1 \mathrm{~mm}$ de diámetro aproximadamente), estas pajillas serán colocadas dentro del soporte de aluminio y se encontrarán en medio de los vapores de nitrógeno líquido, lo que permitirá el enfriamiento.

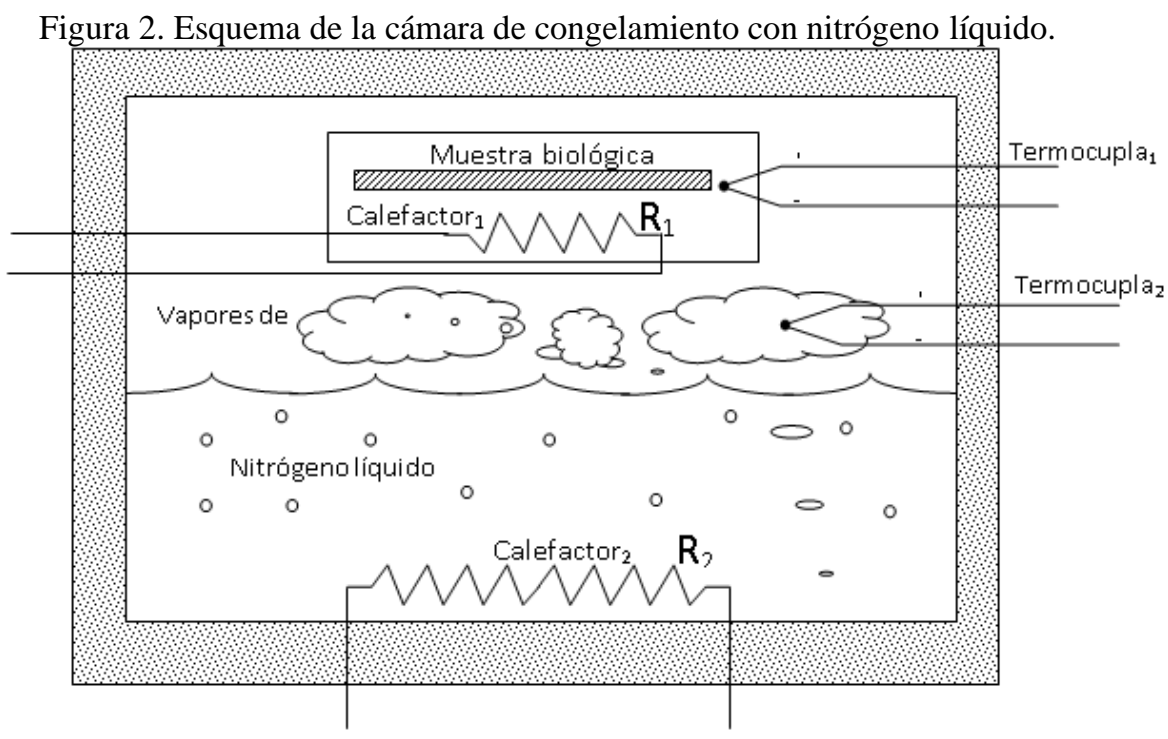

Para poder controlar el enfriamiento, de tal manera que cumpla con el protocolo de congelación elegido, se ha colocado alrededor del soporte un calefactor (calefactor 1 en la Fig. 2) que consiste en un alambre de nicrom, de tal forma que haciendo circular corriente por este cable generará calor suficiente que servirá como barrera térmica a las bajas temperaturas de los vapores de nitrógeno y de esta forma no se enfríe rápidamente.

Figura 3. Vista superior de la cámara de enfriamiento. 


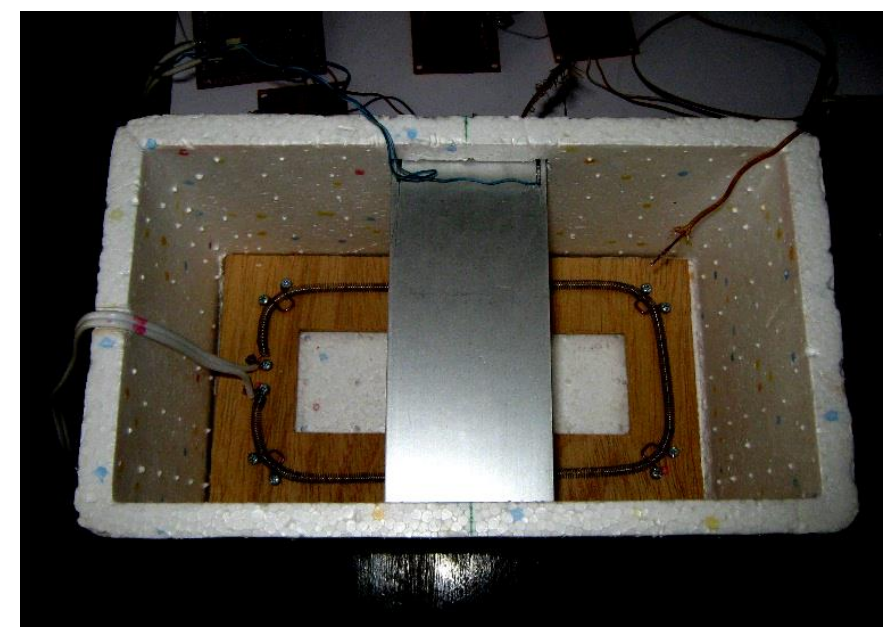

En esta cámara además, se ha colocado otra resistencia (calefactor2, fig. 2) como vaporizador, también consistente en un alambre de nicrom, la cual mediante la aplicación de corriente eléctrica, producirá el calor necesario para la vaporización del nitrógeno líquido, de esta forma se puede incrementar los vapores de nitrógeno líquido durante el proceso, pues este tiende a disminuir conforme transcurre el tiempo, es decir se debe producir mayor cantidad de vapor de nitrógeno si el enfriamiento de los especímenes tiende a ponerse más lento. La temperatura alrededor de la muestra debe estar entre $-80{ }^{\circ} \mathrm{C}$ a $-100{ }^{\circ} \mathrm{C}$ aproximadamente.

\subsection{SISTEMA DE CONTROL}

En circuitos de control que no requieren mucha capacidad de procesamiento y velocidad, son desarrollados con los Microcontroladores denominados PIC, en el presente trabajo debido a la gran capacidad de procesamiento y velocidad, se ha usado un procesador digital de señales (DSP) TMS320F2812 de Texas Instruments, tiene una capacidad de procesamiento de 150 MIPS, y con conversores A/D de 12 bits. Para la programación del DSP se usó el Code Composer Studio v4 (CCSv4), que es un programa que proporciona un ambiente de desarrollo para programar, compilar y depurar los programas realizados para el DSP. El CCSv4 permite también el testeo, la depuración y visualización de resultados.

El control de la temperatura de los especímenes durante el enfriamiento en la cámara, registrará el valor de los sensores de temperatura las termocuplas tipo $\mathrm{T}$ de cobre-constantan, estas termocuplas tienen un gran rango de temperaturas de medición y también fueron elegidos por su bajo costo. En la figura 2 se muestra como se ha colocado un sensor de temperatura en el soporte que contiene a las muestras (Termocupla $)_{1}$ la que irá conectada a un amplificador operacional que acondicionará la señal para la entrada análoga del DSP. El otro sensor de temperatura (Termocupla2), permite controlar la presencia de 
vapor de nitrógeno suficiente para el enfriamiento, va conectado a otro amplificador operacional que acondicione las señales para la entrada análoga del DSP.

Para las termocuplas, se ha construido un amplificador utilizando el amplificador de instrumentación LM725, con salidas de voltaje entre 0 y $3.2 \mathrm{~V}$, que es el rango de las entradas de conversión análoga a digital (ADC) del Procesador Digital de Señales (DSP) TMS320F2812.

En la figuras 4 se muestra el circuito amplificador de las termocuplas 1 y 2, ambas son de similares características, pues ambas termocuplas son de cobre-constantan.

La termocupla 1, es la encargada de detectar la temperatura que debemos controlar y es la de mayor interés pues se encuentra en el soporte que contiene las muestras biológicas, el programa de control en base a esta temperatura, calculará la señal de error respecto a la temperatura de referencia deseada y tomará la acción de control para minimizar el error.

Figura 4. Amplificador para las termocuplas.

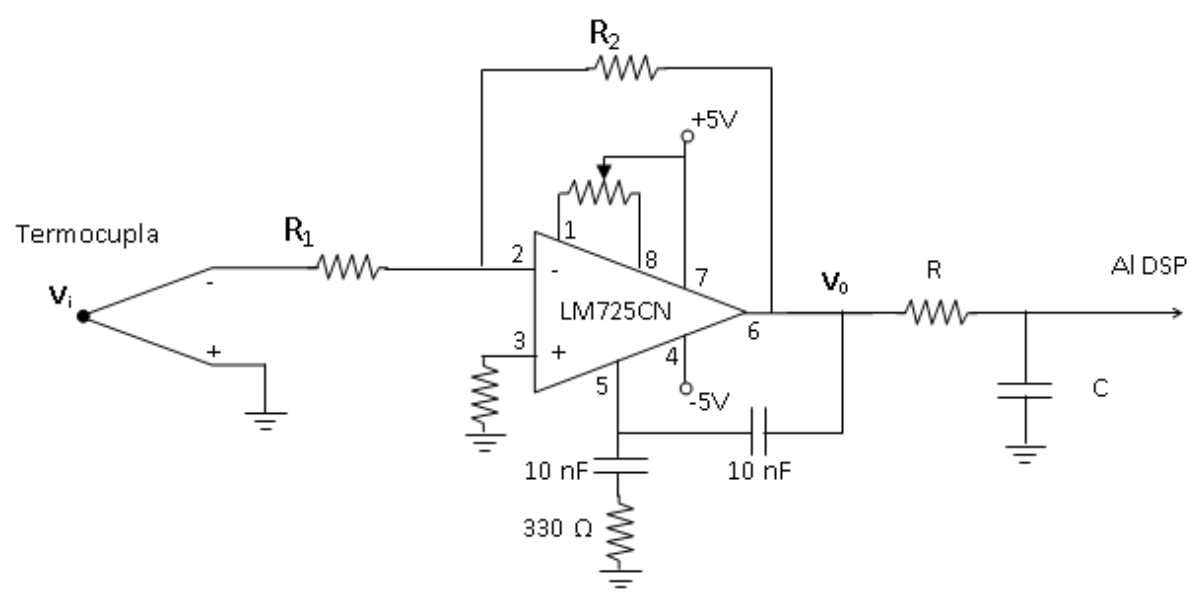

La termocupla 2, detectará la temperatura de los vapores de nitrógeno en los puntos cercanos al soporte que contiene las muestras biológicas, si la termocupla 2 detecta temperaturas mayores a $-80{ }^{\circ} \mathrm{C}$, es indicativo que hay poca generación de vapores de nitrógeno, por lo que el programa de control debe activar el calefactor 2, haciendo que le llegue voltaje para generar más vapor de nitrógeno y bajar la temperatura, este control es de tipo ON/OFF, si la temperatura, gracias a los vapores generados debido a este calefactor, es menor a $-100^{\circ} \mathrm{C}$, el programa ordenara la desactivación de este calefactor.

En los puntos de unión se forman nuevas termocuplas que alteran la lectura de los mismos, ésta se corrigió por programa, midiendo la temperatura ambiental en la juntura de la termocupla y corregir el error de lectura en estas termocuplas sumando esta temperatura a la que indica la termocupla de interés. Se hizo un simple divisor resistivo con el termistor tipo NTC de $10 \mathrm{k} \Omega @ 25^{\circ} \mathrm{C}$ y una resistencia en serie con ella. Como la temperatura ambiental a medir estará alrededor de $25^{\circ} \mathrm{C}$, se ha usado una resistencia de 
Figura 5. Divisor resistivo para la lectura del termistor.

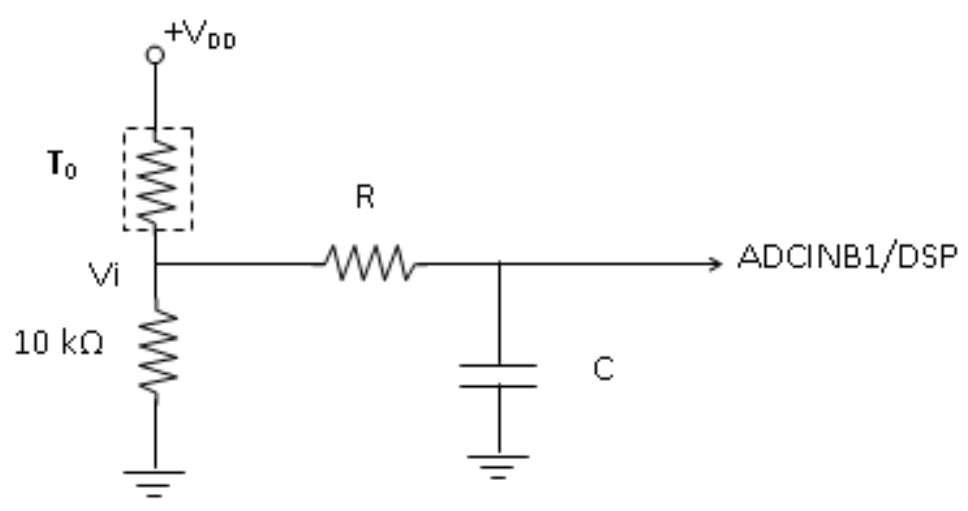

\subsection{ETAPAS DE POTENCIA}

El sistema consta de dos calefactores consistentes en resistencias constituidas por alambres de nicrom, ambas por el efecto joule generarán calor en función de la intensidad de corriente que circule por ellas, pero la finalidad de cada una es diferente, pues mientras una es para mantener caliente a las muestras biológicas, la otra servirá para incrementar los vapores de nitrógeno.

La resistencia calefactora $\mathrm{R}_{1}$ (Calefactor $\mathrm{R}_{1}$ indicado en el esquema de la cámara de la Fig. 2), es la más importante en el proceso de control del congelamiento, esta resistencia consiste en un alambre de nicrom que envuelve al soporte conteniendo las muestras biológicas, el control del enfriamiento gradual será realizado activando este calefactor, de tal manera que si el enfriamiento es demasiado rápido, el calefactor debe dar la energía calorífica para frenar ese enfriamiento, es decir, al envolver a las muestras y dar calor, se estará comportando como una barrera térmica. Este control es realizado por el programa desarrollado para el DSP, haciendo uso del control ON/OFF, el cual estará en función de la señal de error y el gradiente de temperatura de referencia en el sistema. Para esta etapa de control se ha utilizado la salida digital T2PWM_T2CMP del DSP, la cual mediante el optoacoplador fototriac MOC3020 (Fig. 6), activará el TRIAC conectado a la resistencia calefactora 1 mediante una fuente de poder de tensión directa de 70 $\mathrm{V}_{\mathrm{DC}}$. 
Figura 6. Etapa de salida de control de potencia de los calefactores.

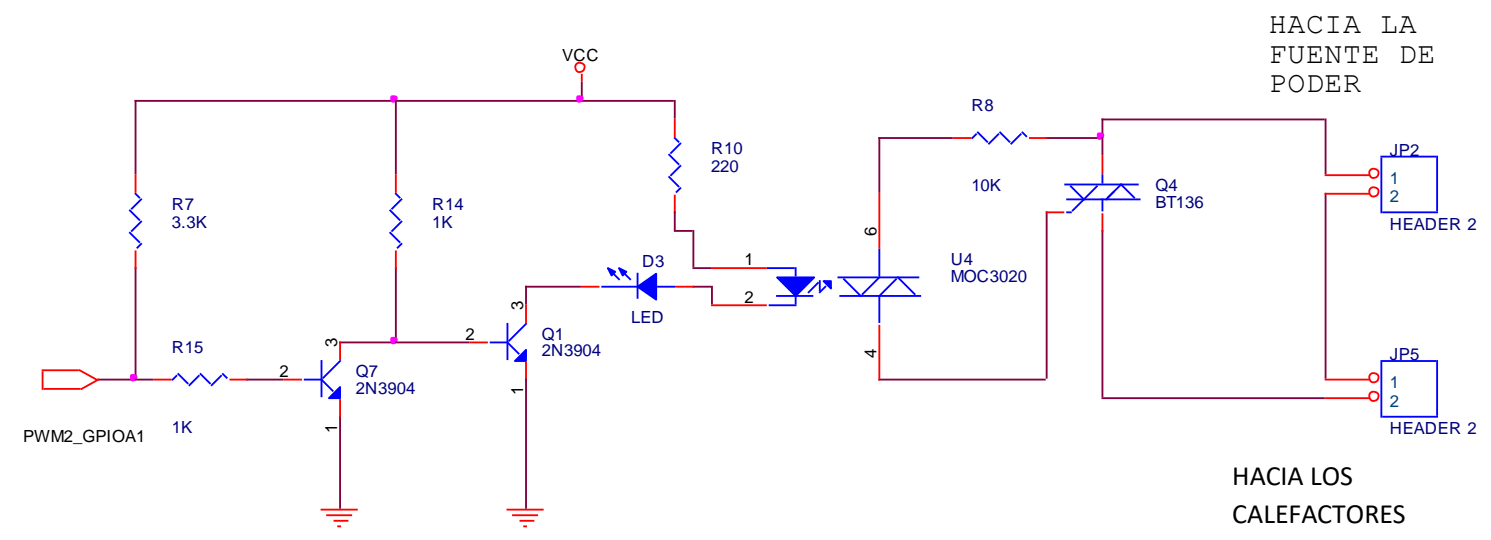

El sistema de potencia de la resistencia calefactora $\mathrm{R}_{2}$ es similar al anterior, sólo se activará en caso sea necesario dar la energía térmica necesaria para la vaporización del nitrógeno líquido dentro de la cámara, si se quiere tener temperaturas mas bajas, es necesario tener mayor cantidad de vapores de nitrógeno, si se le da el calor latente de vaporización necesario, el nitrógeno líquido puede generar más vapores haciendo que la temperatura disminuya en el entorno.

La temperatura en los puntos cercanos a la superficie del nitrógeno (unos pocos centímetros) se encuentran a temperaturas cercanas a $-196^{\circ} \mathrm{C}$, pero centímetros más allá, esta temperatura va aumentando, entonces, en el programa se han incluido instrucciones que estén monitoreando la temperatura alrededor del soporte con las muestras biológicas de tal manera que los vapores alrededor de este soporte esté entre $-80{ }^{\circ} \mathrm{C}$ y $-100{ }^{\circ} \mathrm{C}$, que es una temperatura suficiente alrededor de las muestras biológicas para congelarlas gradualmente hasta $\operatorname{los}-35^{\circ} \mathrm{C}$. La resistencia calefactora 2, es controlada por software mediante un control ON/OFF, a través de la salida digital PWM2_GPIO1 del DSP, esta se activa con un "0" lógico cuando la termocupla detecte que no hay la temperatura suficientemente baja en la cámara, es decir cuando haya una pobre generación de los vapores del nitrógeno líquido, la salida digital activa transistores que mediante el opto acoplador MOC3020, activarán al TRIAC, el que está conectada a la resistencia de nicrom y a la fuente de poder con un voltaje $70 \mathrm{~V}_{\mathrm{AC}}$.

\subsection{PROGRAMA DE CONTROL}

El programa de control de la temperatura, es del tipo ON/OFF, para que cumpla el protocolo de temperatura de la Fig. 7. Inicialmente, se configuran todos los parámetros para el funcionamiento del programa, como son: declarar las librerías, subrutinas y definir las constantes, también se deben declarar las interrupciones, en este caso se hace uso de tres interrupciones: interrupción del Timer0, para el control de base de tiempos, la interrupción del Timer1, que servirá para controlar los tiempos de conversión del ADC. 
Una vez que se da inicio al programa de control, la temperatura para lograr que la temperatura pueda bajar gradualmente desde la temperatura ambiental hasta $-6^{\circ} \mathrm{C}$, se ha comparado la temperatura de la muestra, con la temperatura de referencia esperada, de acuerdo al protocolo de congelamiento elegido; la curva de la temperatura de referencia (Tref 1 en la Fig. 7) en función del tiempo, está dada para el primer tramo, por la siguiente ecuación de la recta:

$$
\text { Temperatura }=\frac{\text { Temp }_{1}-\text { TempAmb0 }}{\text { time } 0} \text { tiempo }+ \text { TempAmb0 }
$$

Se han usado termocuplas tipo T, pues su rango de uso va desde $-200{ }^{\circ} \mathrm{C}$ hasta $370{ }^{\circ} \mathrm{C}$, produciendo una f.e.m. de $-5,603$ hasta $+19,027 \mathrm{mV}$ dentro de ese rango de temperaturas.

Figura 7. Curva de Temperatura vs. tiempo para cumplir el protocolo.

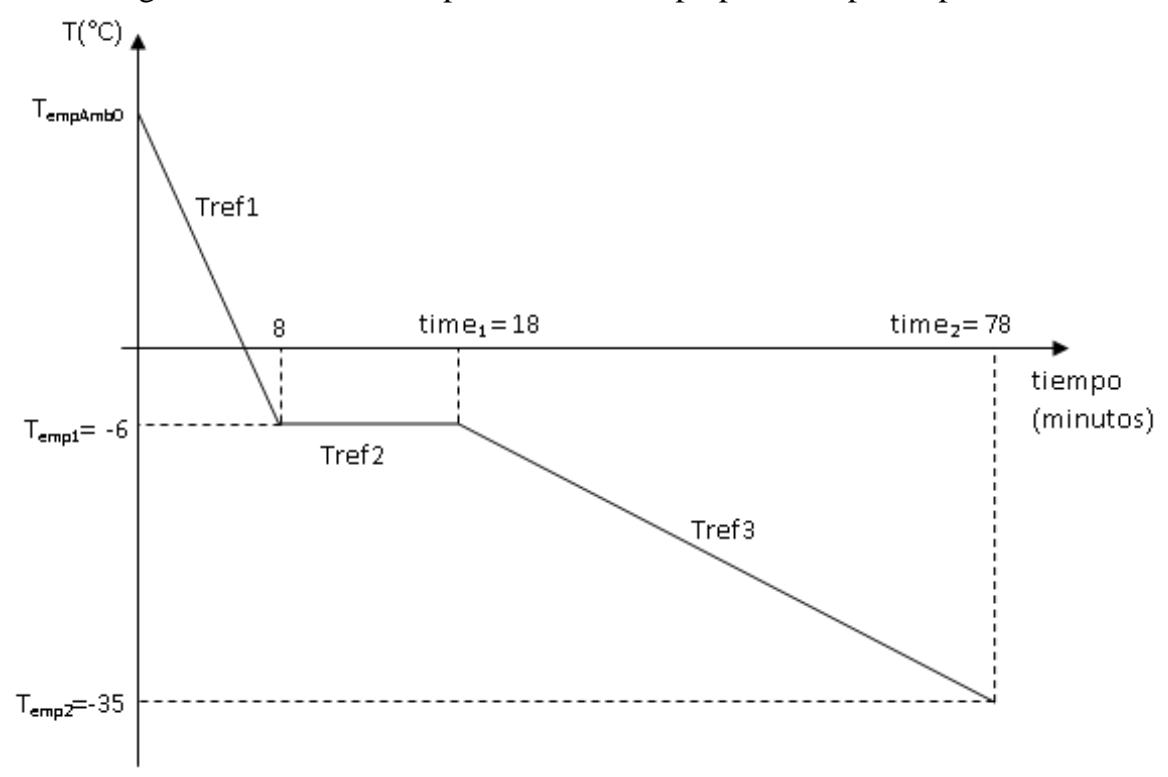

\section{RESULTADOS Y DISCUSIÓN}

En la Figura 8 se muestra el sistema implementado. Los resultados del control de temperatura, fueron registrados por el CCSv4, el cual tiene la opción de graficar en tiempo real, los registros del conversor A/D del DSP. En la Fig. 9, se muestra el resultado de los cálculos del DSP para obtener la temperatura de referencia a lo largo del tiempo. Esta temperatura es la que idealmente debe tener el soporte conteniendo los embriones para su congelación. 
Figura 8. Sistema implementado para las pruebas de enfriamiento

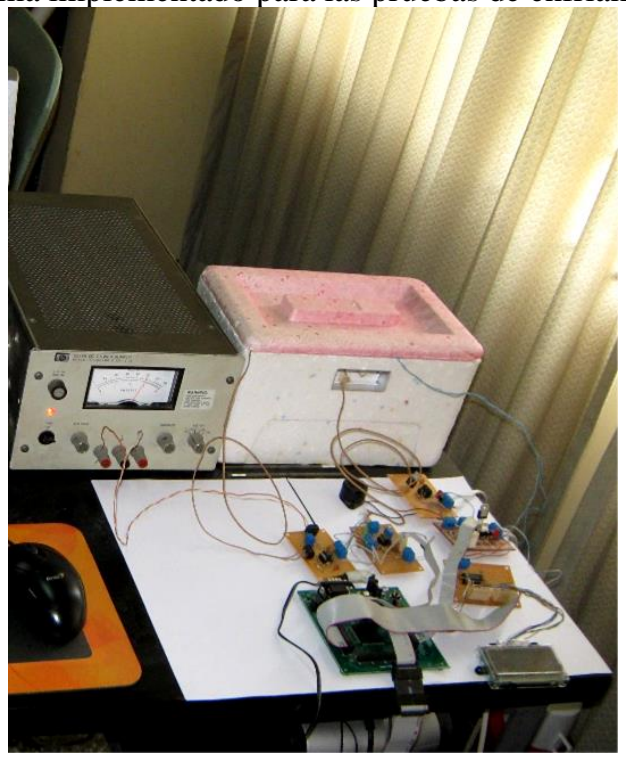

En el eje vertical de la Fig. 9, se muestra la escala de temperaturas (se tiene que dividir la escala entre 100) y en el eje horizontal se tiene la base de tiempo (en este caso no esta en segundos, aquí cada unidad de tiempo corresponde a 5 segundos).

En la Fig. 10, se muestra el gráfico del registro que contiene el valor real medido de la temperatura que se tiene en el soporte que contendrá los embriones, en este gráfico se muestran las temperaturas registradas por la termocupla 1 que se encuentra en el soporte con las muestras biológicas, esta es la señal de respuesta del proceso debido a la acción del controlador, obviando el ruido eléctrico, se puede observar que el control es efectivo ya que no hay mucha diferencia con la curva teóricamente esperada, en este gráfico las escalas vertical y horizontal son las mismas de la Fig. 9.

Se observa que el proceso inicia con una temperatura de aproximadamente a $21,5{ }^{\circ} \mathrm{C}$ y va descendiendo hasta llegar a $-6{ }^{\circ} \mathrm{C}$ en 8 minutos (en el gráfico: 96x5 s = 480 s = 8 min). 
Figura 9. Curva de temperatura de referencia según el protocolo programado.

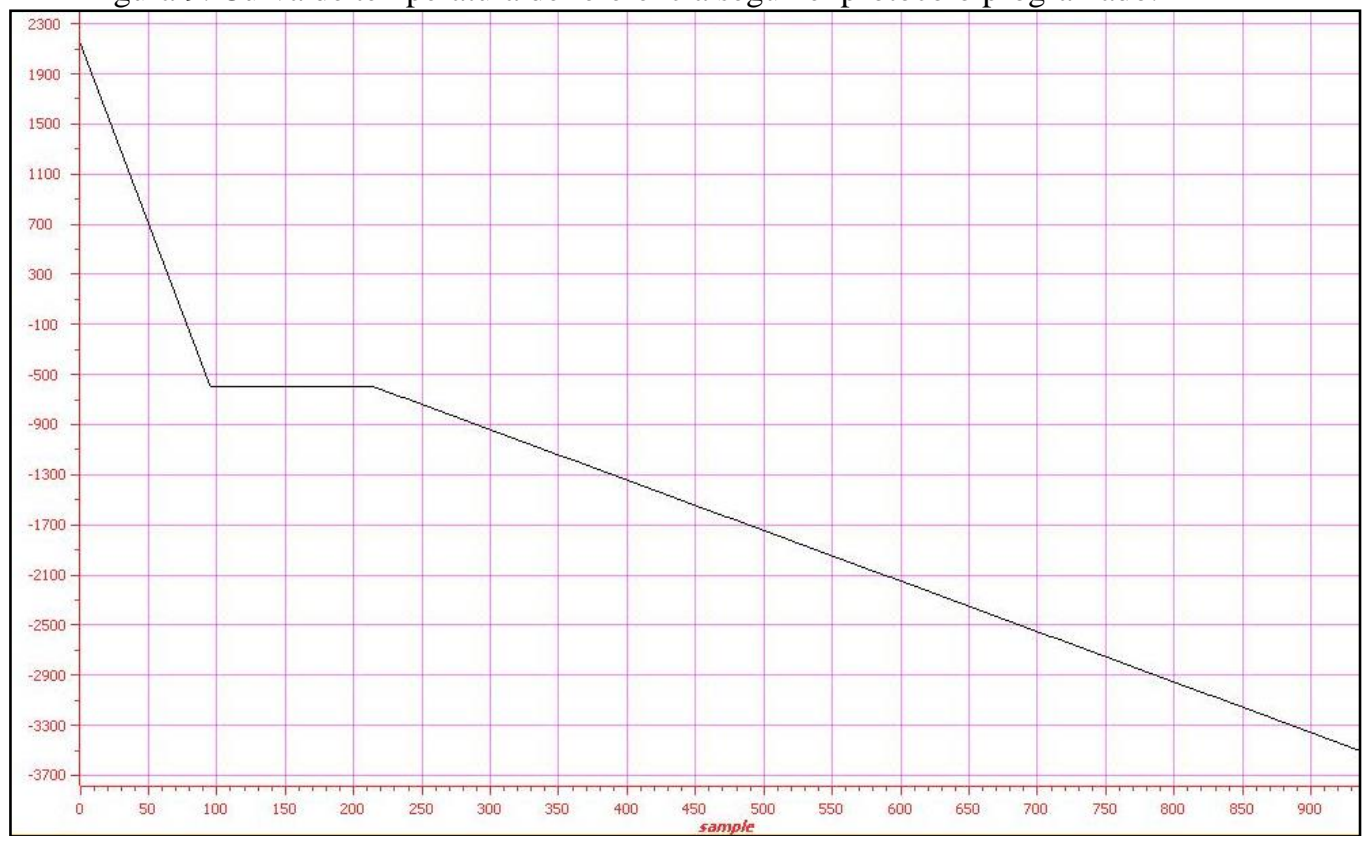

Esta temperatura se mantiene estable en un lapso de 10 minutos (con las divisiones del gráfico sería $120 \times 5 \mathrm{~s}=600 \mathrm{~s}=10 \mathrm{~min}$ ), con ligeras oscilaciones de control de aproximadamente \pm 1 ${ }^{\circ} \mathrm{C}$.Transcurrido los 10 minutos, nuevamente comienza el descenso de temperatura, a una razón aproximada de $-0,5^{\circ} \mathrm{C} / \mathrm{min}$, durante 60 minutos, hasta finalmente concluir en $-35^{\circ} \mathrm{C}$.

Figura 10. Resultado final de la variación de temperatura obtenida.

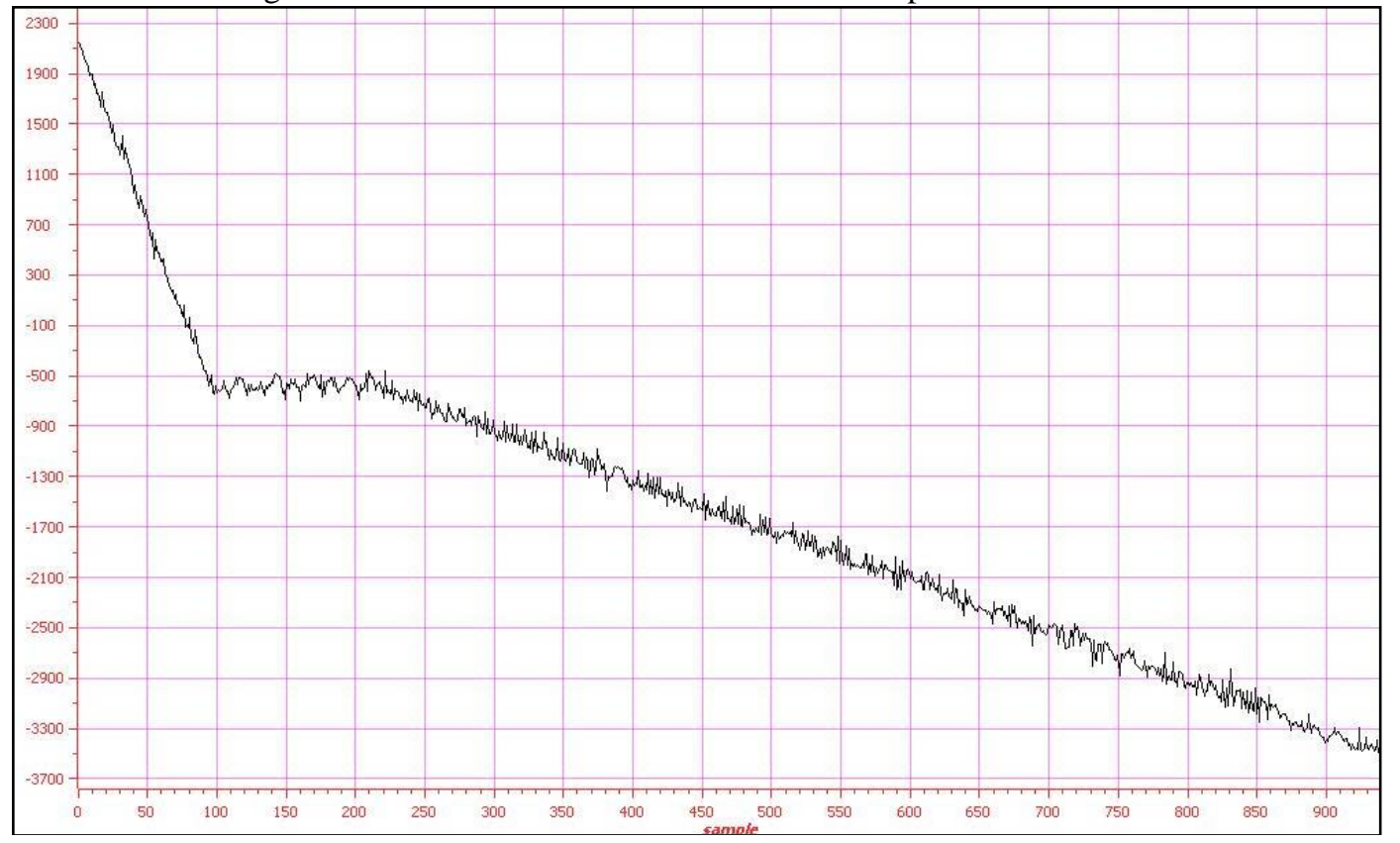




\section{CONCLUSIONES}

De los resultados gráficos obtenidos, se puede ver que el control ON/OFF utilizado en este trabajo fue satisfactorio para el control de temperatura utilizado por el protocolo de criopresrvación de embriones de ovino. La temperatura en el soporte que contendrá las muestras biológicas, graficada en función del tiempo ha ido bajando gradualmente de acuerdo a la curva teórica esperada, y se ha mantenido casi constante en el intervalo de tiempo indicado por el protocolo, con fluctuaciones de $\pm 1^{\circ} \mathrm{C}$ aproximadamente. La gran velocidad y capacidad de procesamiento del DSP utilizado, permitió realizar los cálculos de variación de temperatura en forma rápida para realizar el control. 


\section{REFERENCIAS BIBLIOGRAFICAS}

Fonseca, J.F., Batista, R.I.T.P., Souza-Fabjan, J.M.G., Oliveira, M.E.F., Brandão, F.Z., Viana, J.H.M. "Freezing goat embryos at different developmental stages and quality using ethylene glycol and a slow cooling rate". Arq. Bras. Med. Vet. Zootec., v.70, n.5, p.1489-1496, 2018

Moreano, G., Arregui, C., Tenesaca, C. y Pilco, H. "Rendimiento y eficiencia de distintas técnicas de control en un calefón eléctrico", Ingenius - Revista de Ciencia y Tecnología, N²2 (2019).

Rubio, Luis M. La Criopreservación Embrionaria en la Especie Equina. Medicina Veterinaria 2001; vol 18(9). p 527-546.

Salazar Rivera, Eugenia; López Hernández, Osvaldo; Benítez Lara, Alfredo ; Jiménez Xochimitl, Sandra. PWM controller for thermoelectric devices used in a climatization system. South Florida Journal of Development, Miami, Vol. 2, No. 3, special edition, jul. 2021.

Staples, T.R.; Page, R.D. Freezing bovine embryos with a portable liquid nitrogen freezer requiring no external seeding. Theriogenology, Volume 28, Issue 5, November 1987, Pages 647-659.

Texas Instrumens. Data Manual TMS320F2812 Digital Signal Processor. Literature Number SPRS174S. April 2001. Revised March 2011. 